\title{
Connecting Digitally with the Gen ' $Z$ ': A Phenomenological Study on the Challenges of School Counselors amidst the Pandemic
}

\author{
Monaliza P. Cayatoc MAG RGC, Li Mackenzie C. De Chavez, Casper Dean D. Pineda, Alissa \\ Cyrell T. Cuan, Jordan Louigis D. Roncal, Anroechel Nicole L. Narboneta, Daniel Carlos Tagod
}

\begin{abstract}
Background: School counselors will come across different generations of students with varying experiences and personalities in a counseling environment. A counselor is coming from a more direct and physical atmosphere of interaction, moving to a new digital climate made of more indirect adjustments and changes that may influence the counselor's different experiences towards moving to a new counseling environment. Methods: With five selected respondents, this research follows a qualitative phenomenological approach, using semi-structured interviews to address the challenges school counselors face in interacting with the newer generation of students. Results: This study has revealed three phases found in a cycle of counseling the Gen ' $Z$ ' expressed in the themes: exposure, purpose, and guidance. Conclusion: School counselors face challenges in self-assessment and interaction with the students owing to the digital languages of the Gen ' $Z$ ', whilst maintaining their approaches and ethical standards. Recommendation: Counselors' best practice is the continuous learning of the vast multifarious and dynamic digital languages.
\end{abstract}

Index Terms - Adjustment, Challenges, Digital Language, Ethical standards, School counselors

\section{INTRODUCTION}

Humans are not just physical beings. As such, they not only matter but also imbue them with emotions and passions. It is the combination of these faculties that make up people's humanity. Human movement provides a concrete representation of human emotion and human nature. I seek to portray a harmonious balance of mind, body, and space with honest awareness and sensibility, translating emotion into action. The physical representations can express and clarify inner sentiments (Han, 2013, 8). Given this, it is essential to address their physical needs and emotional concerns.

Moreover, professional school counselors are certified emotional educators and academic consultants with a master's degree in school counseling that trains to support the behavioral needs of all students through a data-driven,

Monaliza P. Cayatoc, MAG, RGC $1,2,3$, Li Mackenzie C. De Chavez 1, 2, 3, Casper Dean D. Pineda 1, 2,3, Alissa Cyrell T. Cuan 1, 2, 3, Jordan Louigis D. Roncal 1, 2, 3, Anroechel Nicole L. Narboneta , 2, 3, Daniel Carlos Tagod $1,2,3$

1. Philippine School Doha, Doha, Qatar

2. Research Development Accreditation and Publication Office, PSD, Doha, Qatar

3. Research Capstone Project, PSD, Doha, Qatar integrated school counseling program that addresses all students' academic, job, and personal or social growth. Counselors challenge themselves to create practical ways and techniques to assist clients in many circumstances. A variety of methods are available to aid in developing client knowledge and, eventually, support the client in moving on in life. However, determine a single approach that works for most clients, so the aim is to be flexible to various clients. The dreams of the guidance and counseling service are similar to the purposes of education in general - to assist the student in fulfilling her basic physiological needs, understanding herself and acceptance of others, developing associations with peers, balancing between controls in the educational setting, realizing achievement, and providing opportunities to gain independence. They are skilled at working in elementary, middle or junior high, and high schools, and they play an essential role in improving students' performance. Having a consultation is a way to help learners become more effective in working with others and help individuals think through problems and concerns, gain knowledge and skills to become more objective, and earn self-confidence. The duties of guidance teachers are critical in effectively applying for the counseling and guidance program at a school, from preparing for counseling and guidance practices in classes to being a model for the students and developing cooperative parents (Gibson and Mitchell, 2003). With these, the guidance counselors vital role in the academic setting emphasizes identifying the students' strengths and weaknesses.

According to ASCA (2020), students in the last six months since September were experiencing stress about the pandemic. Due to that, school counselors have shifted their focus towards online counseling from their regular face-to-face sessions in offices. Additionally, newly introduced programs allow counselors to connect with students even from the confines of their homes. Applications like Zoom and Google Meets are becoming platforms for counselors to be more accessible, even messaging platforms like Facebook and Messenger allow students to have various choices to receive the professional help they need. Consequently, the accessibility of the programs also brings about the problem of privacy invasion and exposure to malicious content and significant risks that will undermine their reputation and safety. Furthermore, other school counselors have difficulty providing required resources and services to students due to pandemic-related challenges. According to the findings of Boudreau (2020), "through survey responses underscored the tireless work school 


\section{Connecting Digitally with the Gen ' $Z$ ': \\ A Phenomenological Study on the Challenges of School Counselors amidst the Pandemic}

counselors and leaders did to continue to support students after the shift to remote learning, counselors also reported feeling as though they lacked structure and direction from leadership to provide personalized support to students."

In alternative counseling, it is essential to note that majority of the counselor association do not use this method and lack knowledge of how this system works. Any treatment that does not fit into the standard treatment technique is considered alternative counseling. It combines the physical benefits of holistic therapy with a more proactive approach to dealing with your mental condition, focused on resolving the issue. Towards the perspective of a counselor, according to Bastemurr et al. (2016), their participant counselors indicate that integration of AT and main counseling theories would increase efficacy. They would not prefer AT because of insufficient psychical environment, ineffectiveness for the long term, problems related to counselors and clients.

This study aims to find out the lived experiences of school counselors given the context of this pandemic, their views towards the new generations, and how the changes in the environment affect their ways of counseling. The topic is shown by the central question: "What are the challenges that school counselors face that affect how they help students?" This situation implies how the pandemic affects their ways of teaching and the struggle of facing a new generation's mindset. The sub-question "How do school counselors manage their ways of helping a student in this generation" relies on the experiences of the selected counselors to answer other questions such as how they maintain work stability through this pandemic and how they learn more about the current generation. This topic aims to examine the views of guidance counselors towards the past generation until the present age, what kind of techniques they implement to understand these students in an academic environment. The aim is to learn more about counseling and guidance understandings and whether these traits differ according to gender, what grade level they handle, and their chosen environment.

The study was able to formulate three major themes: (1) Exposure, with sub-themes such as Challenges, Adaptation and Progression; which focuses on the positive and negative encounters school counselors have faced after the switch from regular counseling to online counseling (2) Guidance, with sub-themes such as Conditions and Coordination; which focuses on the counselors' set of requirements required by the workplace or standards they have set for themselves (3) Purpose, with sub-themes such as Ethics and Approach; which focuses on each counselor's individual mental strength and capability to emotionally guide and support the students no matter the stress of the situation. These are all focused on the categorized experiences of the counselors, specifically, aimed towards the challenges they have encountered and their changes in perspective when communicating with Generation Z.

A healthy human being has spiritual and physical facets. It is also essential that consultation acknowledges a practical way to regain a better sense of balance in people's lives.
Beneficial communication may occur when understanding resonates between the listening parties. This matter, which could not take much place in foreign or domestic literature, maintains its importance as a problem that cannot be sufficiently clarified and is still required to be studied. As was previously highlighted, the performance of an efficient counseling service by the guidance teachers who are the most critical members of the school counseling team obliged to perform psychological counseling and guidance services at the schools has principally relied on their attitude to the counseling and their sense of counseling (Demir and Can, 2015).

\section{METHODS}

\section{A. Research Design}

This study is qualitative and categorized as phenomenological research focusing on seeking the impact on the coping capabilities of School Counselors students and their exposure to the transition of students in social environments due to the advancement of generations. Phenomenology is a qualitative research method used to define how individuals who meet the criteria are subject to a specific phenomenon. It seeks a universal truth from personal experiences, ignoring prejudiced concepts on the responses and experiences concerning a particular phenomenon. The objective of the method is to establish a reliable account of the phenomenon based on the personal accounts of individuals interviewed. It attempts to answer two crucial concepts, the experience itself and the elements that influenced the experience's impact. According to Remeyni, D., Williams, B., Money, A. \& Swartz, E. (1998), Phenomenology in business research focuses on experiences, events, and occurrences with disregard or minimum regard for the external and physical reality. Phenomenology, also known as non-positivism, is a variation of interpretivism and other variations such as hermeneutics and symbolic interactionism. This branch of philosophy "describes the philosophical approach that what is directly perceived and felt is considered more reliable than explanations or interpretations in communication.

A research involving an extensive understanding of moral and ethical connections of the counselor's challenges which are referred to as the phenomenon and serve as the focal point of the study. A thorough investigation and understanding to conclude the research and prove or disprove the researcher's assumptions through a refined analysis. The research connects perspectives and objectives from different individuals on the specific results of the challenges to expound on the views of the individuals towards the phenomenon, the challenges school counselors face, and how these affect their performance. 


\section{B. Data Collection and Ethical Consideration}

The researchers used two elements to capture the nature of the phenomenon, the first of which is the robofoto, a personal data sheet of the research respondents that includes vital professional and confidential information that requires the demographic sketch of the participants, precisely their amount of experience in the counseling field. Participants were handed the robofoto before taking the interview. The second element used was a twenty-five-item quasi-structured interview; in the interest of a more in-person interview, it links to the assumption that the participants to be interviewed viewpoints would express better than they would be in an interview without facing one another - this kind of interview conducted by the researchers upon the approval of the participants through their written consent. The opportunity to observe the discussion while reading a transcript, advancements technologically present the lightening of the pressure on note-taking while the recordings allow one to inspect the data better.

The interviews are set on the counselors' time since some respondents live in different time zones. Take precautions and make sure that both the respondent and the interviewer are relaxed and free at their time with no further distractions unless there might come an emergency and have to delay, then there will be another arrangement for it if the respondent agrees. Before the recording begins, the counselors will be reminded at the very beginning of the video call that they will record and if they will be comfortable with it or not; the interviewer will reassure the respondents that the interviewer will not post the recorded files on any social media platform that researchers will only use this in the study. The researchers did not do the meeting for the interview in person due to safety purposes, and we have planned to interview the respondents on a virtual platform of their choice of video call website or what most online classes use currently.

It is best to give respondents the utmost anonymity and confidentiality in research endeavors concerning interviews, questionnaires, and the likes. The Belmont Report supports these principles, created in protecting human subjects in research. To preserve the anonymity of the participants, the researchers took several precautions to keep the identities and information of the respondents hidden. They are blacking out their names completely in transcripts and replacing them with more unattributed callsigns (P1, P2...). In pursuit of anonymity, personal information divulged by the interviewees, keeping their personal information under lock and key and not letting anyone who has not authorized view the file in any way, shape, or form.

\section{Research Locus and Sample}

The study was conducted adequately in Philippine School Doha, Qatar. The researchers selected this location because this is where they have planned their research and that there were already participants that are available in their alma mater, though the researchers had to find more participants for their study, so they asked other schools in Qatar. Unfortunately, due to the lack of responses, the team contacted other schools outside of Qatar to give consent to interview their counselors. The chosen respondents for this study are school counselors who have worked in the field physically for more than four years but are currently running digital counseling sessions for this study's duration. Due to each individual's specific experiences, the validity of the analysis may expand by choosing a small but diversely represented number of cases. According to Boddy (2016), sample sizes involving even one point can be highly informative and meaningful, as demonstrated in management and medical research examples. Furthermore, triangulation has its origins in attempts to validate research findings by generating and comparing different sorts of data, and other respondents' perspectives, to achieve significant respondent validation (Torrance, 2012).

\section{Data Analysis}

The primary source of information came from the verbalizations of the participants. The first level of the data is in the following steps: (1) through a semi-structured interview, analyze the participant's responses. Taking notes of what is said and transcribing for better analysis; (2) thorough examination of the transcribed data, and cleaning up of the answers (emic) and understanding the respondent's interpretations according to the context of the questions; (3) translating the ideas of the interviewees and understanding of the researcher; (4) organize the similar responses of the respondents and sort them into themes via a dendrogram; (5) create a visual representation of the findings via a simulacrum.

\section{RESULTS}

Counselors are known to be adaptive and observant, though the digital side of counseling proved to be a challenging setting to move into. The simulacrum pertains to the cycle and phases of counseling the Gen ' $Z$ ' accompanied with the approaches a counselor uses to adjust and work to interact with their clients. There are three themes extracted from the research - Exposure, Guidance, and Purpose. After switching from regular to online counseling, the first stage shows school counselors' exposure, learning the digital view and the new environment. The next phase focuses on finding purpose, and how a counselor's mental strength and capability emotionally guides their client's stressful situation, not to mention the pandemic.

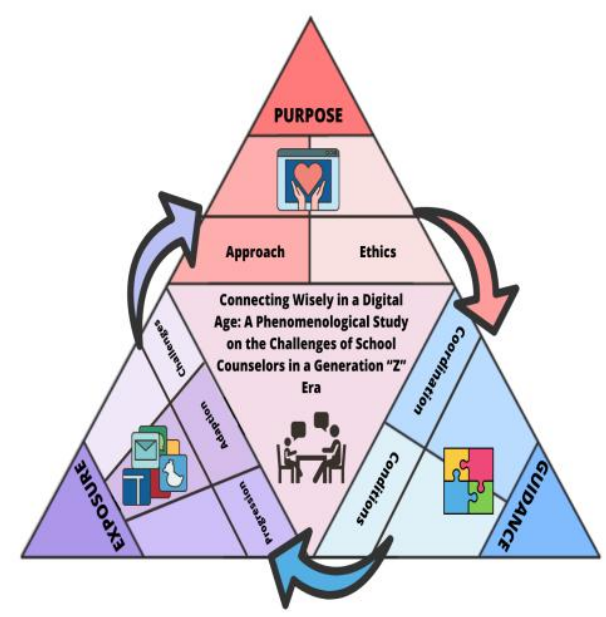

Figure 1: The Cycle and Phases of Counseling the Gen ' $Z$ ' 


\section{Connecting Digitally with the Gen ' $Z$ ': A Phenomenological Study on the Challenges of School Counselors amidst the Pandemic}

The symbols placed in the middle of each theme represents the themes themselves, the character for the exposure being social media applications that counselors use to connect with their clients, the puzzle pieces of purpose to show how the counselors' experiences to understand a client's viewpoint, and the symbol for guidance representing the importance of their standards and proper communication. If the triangle's three stages are unidentifiable, this will not appropriately deliver the representation of the guidance counselor to the learners. In addition, the arrows shown denote the cyclical nature of the three phases Exposure, Purpose, and Guidance.

\section{Exposure}

A guidance counselor's job descriptions are known to be around face-to-face interaction, but in this new setting during the Gen ' $Z$ ' and pandemic, this will be a progression that needs to be studied. As the environment changes, their clientele would prefer the more convenient online interaction rather than the face-to-face counseling the counselors used to have. With the newer generation of clients being more inclined to online interactions, guidance counselors must now act accordingly and cater to them through online counseling while maintaining the same procedures and standards they hold during face-to-face meetings. In this stage, counselors begin adapting themselves to the digital world, giving them a new perspective on their clients' faces. Counselors begin to adapt using what is accessible around them, using technology to be still able to communicate with their clients while still following their procedures with the a respondent realizing;

\section{“...But I have difficulty with the digital languages because I} am not familiar with it." (P1)

Being immersed in the digital world, the counselees utilize slangs that may not be understood by the counselors. Counselors need to adapt to the digital language to better improve connections and communication as observed by the respondent,

"My generation has some similarities with the current Generation Z. They are very fast when it comes to technology, they are very knowledgeable with digital things. If you want them to understand you, you have to learn their digital language." (P2)

During the pandemic, counselors relied on using the digital language to adapt in order to communicate with each other. Counselors do not only interact with their clients but with their colleagues as well to see the similarities and differences. A respondent noted:

"In this pandemic situation, we are used to things face to face. In my mindset, I must accept what is happening around me and get used to handling my students virtually through Tele counseling/teletherapy.” (P4)

With the preoccupation of their clients towards online communications, counselors move on to those online platforms correspondingly to further make themselves accessible. Because of the pandemic, their newer clients contrast with their older counterparts, who prefer face-to-face counseling. As articulated by the respondents,

"Due to the significant changes in the environment, the students are more fixated on texts, email and zoom and that is what kind of counseling I give to them. Although generations before would prefer to come in and talk face to face." (P3)

Counselors realize the challenges slowly as they adapt to the new environment; they can recognize the negative and positive aspects of the digital world. Even with the issues present, it is still manageable wherein both the counselor and client work together to avoid future problems that can affect the client's reputation and choices. As understood by the respondents,

"This generation is caught up in social media and what people are saying about them both physically/virtually. One of the biggest challenges with this generation is trying to get them to sort through all those problems responsibly and respectfully." (P3)

The comfortability of a client is the key for counselors to form their sessions in a specific environment, but when it comes to a generation that counters their sessions, it draws a setback between the counselor and client relationship. As they look into the new generation's environment, this action helps counselors find better ways to ease new clients that are new to the counseling environment. Providing a warm welcome and proper atmosphere to help them open up, they slowly interact and analyze this generation's situation as they begin to use what is advantageous around them. As mentioned by the respondents,

"The nature of interaction in the counseling atmosphere might be challenging because generation $Z$ 's are very informed. There are times we self-diagnose but we have to be more open minded." (P1)

"We must establish trust for us to assist them, otherwise they might self-diagnose from the internet. We need to develop acceptance, develop understanding, and empathy, we have to deal with some issues by heart." (P1)

In the pandemic setting, counselors face the challenges of the digital environment. As they utilize their clients' platforms for messaging, they continue to try their best to adapt to the current situation as health does not wait for anybody. Though the new working environment is unique to everyone, thus providing many setbacks to those who are not too familiar with the virtual world, counselors make sure to view the situation thoroughly and recognize the advantages of using technology as a medium for counseling. Even after the pandemic, counselors can rest assured that they have gained valuable experiences and learned new things to incorporate into regular counseling.

\section{Purpose}

Every operation a counselor faces teaches a new understanding of a client's perspective. Guidance counselors set themselves ethics depending on what situation presents 
themselves. They create their objectives while also familiarizing themselves with their client. There are many stages that a counselor has to go through to grasp what techniques they should use. During the purpose stage, counselors use their talents to observe any changes made during the consultation to develop a proper resolution. As the respondents have stated,

"Counseling requires me to listen very carefully and to assist very carefully. I see how they're functioning, their word choices. that gives me a really good idea of where they are in terms of mental health and wellness." (P5)

Before counselors meet their clients, they do a background check to see what interests them and affects their health physically and mentally. Counselors find it essential to have understanding conversations to connect with their clients. As the respondents have mentioned,

"When it comes to attitude, students tend to feel hesitation and it takes time for them to open up. The difficulty here is that we need to assure them with full confidentiality for us to understand." (P4)

"In terms of age, it is part of your task to learn what the youth is interested in. You have to be familiar with what they know to make a proper connection." (P1)

"You need to know your counselee first before you do counseling. It is important to know where the student came from, their background and interests, what are the things that these students are engaging in their past." (P1)

Counseling requires a set of traits to improve their consultation process, but what they consider the most valuable is what kind of therapeutic technique they should use to start a session. Techniques used in counseling are essential in data gathering, helping counselors form answers and investigate further what they can do for their clients. As the respondents have disclosed,

"We conduct homeroom guidance sessions for students, which gives them information to have an idea on the discussed topics. We make use of the REBT(Rational Emotive Behavioural Therapy) technique." (P2)

There are certain limitations in counseling, especially when guiding clients emotionally. The act of placing personal prejudices and constraints on clients in therapy is discouraged. Counselors aim to help clients work through their problems, not to tell them what they should and shouldn't believe or do. As the respondents have expressed,

"I tend to draw from a variety of different theories and approaches. Every session is tailored specifically that there are lots of reasons why we have very negative experiences that students have. We help the student work through those things in a manner that works for them." (P3)

"There may be scenarios like the word transference, this means a client's situation is transferred to the counselor's perspective, that is why we must be careful with our words.
We are trained professionals and we must be prepared at all costs for any situation." (P4)

"We have to be mentally, physically, and emotionally disposed when counseling. We have to maintain our objectivity and our empathy. We can't be sympathetic in our counseling, because we cannot be an effective counselor if we invest our emotion into the issue." (P1)

Even with the difficulties of how counselors manage, they still familiarize themselves with their procedures and objectives. With all the responses given, it is evident that counselors are careful in making decisions based on how many methods they need to follow to provide an answer that opens to more ideas. Whether the situation is complex, counselors train to handle it using their experiences and studies to investigate what to fix. Clients should note that counselors have different backgrounds and ethics to a situation.

\section{Guidance}

When it comes to student counseling, they have different conditions before having open and honest communication. Meanwhile, during the counseling, the counselors will keep their word not to spread their discussion and maintain confidentiality. They guide the student throughout their conversation and make their client understand themselves more. Although there are different approaches to each school level that the counselor gives, they have to manage a calm demeanor to their client. Some situations that counselors face will be more complex than manageable in the coming meetings, giving them a new aim to add to their operation. When it comes to violent reactions or self-harm, they can inform their parents about the student's well-being as part of their protocols to ensure safety. As experienced by the respondents,

"There are certain cases that you need to inform the parents, especially if this involves hurting oneself. There are only four or five instances where we cannot keep the confidentiality and we need to tell somebody for your safety and welfare." (P1)

"I would not approach family members out of concern since my practice is different. For me to approach a family member, they would have to get explicit written permission and this should be respectfully open and honest. This is to remind them that we are handling this at a point of concern." (P3)

When a client asks for counseling, there are different ways to raise awareness for family members. A few counselors will offer the client's parents written permission to see if they are willing to support and agree with this arrangement for their son or daughter. As noted by the respondents,

"I would not approach family members out of concern since my practice is different. For me to approach a family member, they would have to get explicit written permission and this should be respectfully open and honest. This is to remind them that we are handling this at a point of concern." (P3) 


\section{Connecting Digitally with the Gen ' $Z$ ': A Phenomenological Study on the Challenges of School Counselors amidst the Pandemic}

A counselors' responsibility is to create a safe space for their client to open up. Connecting with the client and communicating with them gives them a diverse experience that suitably caters to each client's needs and guides them through the process. As disclosed by the respondents,

\begin{abstract}
"In my type of counseling, this is all about helping a person be comfortable. My methods is that we have to speak in a very safe environment and I have to adjust to these students for them to open up. I ask them how they would want to be guided, and from there we communicate." (P5)
\end{abstract}

In a counseling relationship, the client should feel comfortable sharing. To achieve this, the counselor has to ensure that the counseling environment is a safe space for the client. Furthermore, the counselor needs to go at the pace of their client and give them a sense of control to help reinforce the idea of security in counseling. Counselors play a significant role in being flexible and culturally responsive to create an atmosphere that suits their clients more effectively.

\section{DISCUSSION}

Counselors use their developed abilities to form new ways of thinking to view different perspectives. According to the American School Counselor Association (2019), a school counselor's role focuses their skills, time, and energy on direct and indirect services to all students, regardless of their citizenship, national origin, race, color, gender, sexual orientation, socioeconomic status or any other demographics. School counselors participate as members of the educational team and use the skills of leadership, advocacy and collaboration to promote systemic change as appropriate. Supporting all students with various needs may include a diverse skill set, including knowledge about many legal factors affecting students. It shows that counselors are diverse in training and experiences on how they formulate solutions.

Furthermore, in the digital environment, safety applies to the online platforms they use to conduct online counseling. Counselors are also to raise awareness of what happens inside and outside of social media; this alerts students to be more aware of what they search and post. According to the American School Counselor Association (2017), school counselors find that technology may help find academic opportunities while students need to be proficient in the use of technology. However, when students access web-based and mobile technology, they leave a digital footprint that makes them vulnerable to privacy invasion. They expose themselves to harmful content and significant risks that compromise their safety, security, and reputation.

Moreover, a cycle of a counselor's methods comes with different aspects from experiences and teachings, considering the influences made during consultations. It will further link to how the operation, ethics, conditions, coordination, progression, and adaptation affect the process of confirmation into how counselors consult the new generation and environment.

\section{Exposure}

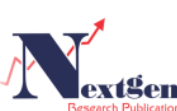

\section{Challenges}

Counselees of the new age are brought up in the digital world and have, in turn, absorbed the slangs and languages of the modern era. Especially with the rise of social media applications, the use of slang is more prevalent than ever. Adolescents and young adults now predominantly use social media and other digital channels to connect, develop relationships, and stay informed. It isn't just for kids, though. People of all ages use the internet to stay in touch, follow their passions, and form new relationships. Counselors who ignore all conversations or don't have a basic comprehension of social media risk alienating their clients and failing to interact with them (Laura Gallo 2014). Counselors then are responsible for building a connection with their clients through the acknowledgment of these informal languages. School counselors must not only be able to use technology, but also have a basic understanding of digital literacy. According to a study done by Limilia \& Aristi (2019), The quality of e-resource use is strongly related to basic digital literacy. This means that someone will be able to comprehend and apply information in a variety of formats emanating from a variety of digital sources presented on computers, and e-resources are information sources that require computer devices that can be accessed both offline and online. As a result, counselors can utilize social media to their advantage regardless of whether it is used to engage with their clients or work with peers, though many counselors are unfamiliar with the platforms. As explained by Gallo (2014), counselors must strive to comprehend this new culture, which has its own language, values, and customs. If a counselor does not identify as a "digital native," they may be unaware of the complexities of this culture and find it difficult to recognize its significance in their clients' life. Counselors who make an effort to understand this ethnic group are more likely to sympathize with and connect with their clients, strengthening the counselor-client relationship. The majority of counselors see how technology has become ingrained in our daily lives. Accepting what it means to a client may be more important than whether or not it is welcomed or cherished.

\section{Adaptation}

The frequent use of technology in students' lives necessitates a focus from adults to understand its use and impact. This trend involves school counselors keeping up with the changes in educational technology, critically evaluating the available solutions, and implementing those that help them accomplish their duties. Digital natives have created a new culture with language, values, and customs. Digital immigrants may not always be aware of the intricacies of this culture and struggle to accept its importance in students' lives, yet, as they would for any cultural group, school counselors seek to understand so, they can be empathetic to students' interests and help create positive change (Gallo, Rausch, Smith, \& Wood, 2016). Technology tools that help school counselors communicate and present information through technology address issues of efficiency by automating and digitizing delivery and help them reach more students, families, or staff (Cleveland \& Sharp, 2019). Discussions of the use of technology in school counseling increasingly focus not on the 
technology itself but on the promise of technology as a delivery mechanism to increase the effectiveness of comprehensive school counseling programs (Steele, Nuckols, $\&$ Stone, 2020). This knowledge would well serve school counselors to grasp how technology might assist their job since they play a crucial role in influencing students' learning, communicating, and sharing knowledge with others. According to Laura, Timothy, and Russel (2008), when it comes to finding more efficient manners in providing quality services, the appropriate use of current and emerging technology is one viable solution for dealing with this problem. The necessity for a comprehensive and integrated approach to counseling, including accountability, has become increasingly necessary, especially as fewer counselors are working with increasing numbers of clients in all sectors. With online learning being relied on in recent times, students enroll in virtual schools, which range from hybrid, which utilizes a balance of online learning and face-to-face environments, to fully online classes (Holmes \& Kozlowski, 2016). As the counselors meet the digital world, they understand the challenges of the new environment, like digital counseling limitations. Furthermore, they have to continue practicing the same standards and procedures as they would in a face-to-face setting (Wilczenski \& Coomey, 2006). Online counseling aims to reach a diverse set of clients that they will not receive in a traditional face-to-face environment.

\section{Progression}

To understand the client is to understand their language. According to Chibbaro, Ricks \& Lanier (2019), as digital integration into society continues to emerge at increased rates, school counselors must stay abreast of current research and trends within this area to address the needs of their students' needs community. Counselors realize that their clients are heavily dependent on technology, causing difficulties that affect them in various ways. For example, the learners struggle to express themselves fully because they feel anxious in the new environment, or the student cannot fully express themselves due to the limitations that online counseling presents (Weinberg \& Rolnick, 2020). Additionally, many students experience difficulties with identity formation. According to Flum and Kaplan (2006), it helps counselors help their clients to be more adaptive, attentive, and self-sufficient in making critical decisions while also developing a strong sense of competence, providing learners with the feeling of safety, identification, and connections, helping them grow academically, psychologically, and social development (Jethwani-Keyser, 2008). Often these events can be stressful and highly challenging for students to cope with and understand. To mitigate this, the formation and enhancement of counselor-client relations can, at the minimum, help ease the transitions and difficulties many students experience (Wigfield et al., 2005). Furthermore, counselors assist with school-related issues and personal issues that may be affecting the client. Students who receive sentiments of support and encouragement from a counselor, teacher, or mentor are more likely to become receptive, motivated, have higher self-esteem or ability to manage, and become more actively involved.

\section{Purpose}

\section{Approach}

Counselors use multiple therapeutic processes to determine how they will be able to help their clients. A study conducted by Wigfield and Wagner (2005) found that when appropriate relationships are established between counselors and clients, counselors can ease client transitions and difficulties. Specifically, in a school setting, the developmental needs of many students can also be supported by this type of counselor connection and can also assist with clients feeling supported (Feller \& Cottone, 2003). Counselors make moral and ethical decisions based on various systems, including their morality theoretical understanding, organizational policies and procedures, and an ethical framework for counseling. One of the mentioned therapeutic processes is Rational emotive behavior therapy (REBT); according to Crystal Raypole (2018), it is an approach that helps you identify irrational beliefs and negative thought patterns that may lead to emotional or behavioral issues. This theory shows that the different styles used in therapy sessions can create different outcomes depending on the client. From theory and research, in general, guide counseling techniques, both of which shape the way of practice. These may be conditional on how counselors communicate with their clients and distinguish between counseling and coaching designs and styles. The objective is to help clients acquire the psychological tools to deal with difficult emotions and situations as to their current situation and environment. Clients learn that their psychological difficulties connect to environmental stresses throughout the Interpersonal Counseling. In this case, Interpersonal counselors are helpful and sympathetic assisting their clients. Markowitz \& Weissman (2004) suggests that counselors advise clients on how to deal with problems to increase self-efficacy and decrease symptoms, as Interpersonal Therapy has shown to help treat mental diseases, particularly depression, in clinical trials. Most cases emphasize the necessity of letting the client make their discoveries rather than giving the counselor much guidance resulting in a Humanistic approach to counseling. As a result, when working with a client-centered counselor, the client often conducts most talking.

Humanistic counseling assumes that people already have the skills they need to succeed. According to a study, this strategy promotes curiosity, intuition, creativity, humility, empathy, and charity. (Giorgi, 2005; Robbins, 2008). Client-Centered counseling creates a safe atmosphere with a sympathetic and non-judgmental therapist. As a result, the client will feel a sense of acceptance, openness, and unconditional positive respect. After establishing Humanistic Counseling, a Humanistic Counseling technique helps clients reach their full potential as human beings.

By revealing genuine functionality, a counselor can better understand behaviors. Cognitive-Behavioral Therapy focuses on how a person's feelings influence their ideas and behaviors in a specific scenario. According to Hoffman \& Smits (2008). Cognitive-Behavioral Therapy, based on the idea that "cognitive variables perpetuate emotional illnesses, and psychological treatment leads to improvements in these factors through cognitive and behavioral strategies." Many of the counseling modalities mentioned address trauma and loss; several are particularly effective.

Ethics 


\section{Connecting Digitally with the Gen ' $Z$ ': A Phenomenological Study on the Challenges of School Counselors amidst the Pandemic}

Counselors should monitor the frequency, duration of contact, types of topics discussed, and location of interactions to ensure each category's appropriateness. They watch the potential impairment of their objectivity that may lead to the exploitation of students. Holcomb-McCoy (2004) also found evidence supporting the need for school counselors to understand the impact of culture on the counseling process. By reference, several factors have influenced the growth in online counseling: an increase in technological developments, the establishment of online counseling training programs for practitioners, and the development of ethical guidelines for online counseling by professional bodies (Barak et al., 2009). According to Cohen and Kerr (1998), "online or cybertherapy has gained substantial scrutiny and acceptance as a reasonable counseling conveyance approach" over the last two decades. According to a study, online counseling is just as beneficial as in-person counseling for treating anxiety and depression (Andrews et al., 2011; Wagner et al., 2014). In online counseling environments, therapists tend to reflect more to convey empathy, strengthen therapeutic communication, and improve the quality of their interventions utilizing online tools and techniques, consequently improving their listening, writing, and attentiveness abilities (Anthony \& Goss, 2009; Owen-Pugh, 2013; Tsalavouta, 2013). It showcases how the counselors will open up and improve their skills as they are introduced to online counseling and learn more studies. When treating specific mental health issues, the emotional connection between the therapist and the client in online counseling is either reinforced or absent, altering the therapeutic process (Tsalavouta, 2013). Members stated that secrecy, comfort, and the pastoral or spiritual counselors' or psychologists' qualifications are the primary reasons for seeking online counseling. Kanani and Regehr (2003) and Young (2005) conducted an overview of those who had expressed an interest in online chat sessions for compulsion issues and found that members declared secrecy, comfort, and the spiritual counselors or psychologist's qualifications are the primary reasons for seeking online counseling. Counselors' awareness of student differences in language, values, attitudes, norms, and customs are crucial to consider when building a client-counselor connection. Language, value differences, and prejudice may lead to less than accurate information obtained during the consultation process.

Guidance

\section{Conditions}

Using the current environment and creating a new one for a client helps counselors avoid miscommunications and help develop a better understanding of a difficult situation. The benefits of building trust, genuineness, caring, empathy, multicultural competence, and awareness for all students also result from the understanding between counselor-client connection are the students' feelings of support and encouragement, assistance in easing transitions and challenges, and academic and social progress (Feller \& Cottone, 2003). School counselors are well-versed in the benefits of a secure, organized, and compassionate learning environment. Students gain communication, problem-solving, and conflict-resolution skills that help them achieve their goals and build successful relationships through participation in anti-bullying, anti-harassment, and violence prevention programs and activities. A secure, supportive, and fair learning environment involves providing physical and emotional safety structures. In the given environment, school counselors are still to deliver school counseling services through a virtual or hybrid setting including the three domains; School counselors provide programs that have an impact on student growth in three domain areas: academic development, career development, and social/emotional development (ASCA, 2019). After schools closed last spring due to the pandemic, attendance and discipline data may not have been collected or viewed differently than in previous years. In that case, achievement data may be the only source of outcome data to understand student needs. School counselors can also use supplemental data such as needs assessments and program evaluations to gain greater context about the outcome data. Data obtained from these assessments can be analyzed and reported to school administrators, faculty and parents in ways that highlight the current program's accomplishments and limitations. Findings from these reports will provide the evidence needed to determine whether all three school counseling domains address the virtual or hybrid setting (ASCA, 2020).

\section{Coordination}

Counselors always ensure that their clients, whether students or adults, are in a secure environment and have followed their protocols. BACP (2018) stated that counselors' endeavor to demonstrate equality, value diversity, and ensure inclusion for all clients. They take the law concerning equality, diversity, and inclusion into careful consideration and strive for a higher standard than the legal minimum. Counselors use confidentiality as the ethical and legal phrase to describe the information shared in a therapy relationship, and counselors must preserve it unless keeping the information private would cause predictable harm. Trust is the cornerstone of the counseling relationship, and counselors have the responsibility to respect and safeguard the client's right to privacy and confidentiality (ACA, 2014). Another statement by American Counseling Association (2014) states that counselors recognize the need to balance the ethical rights of clients to make choices, their capacity to give consent or assent to receive services, and parental or familial legal rights and responsibilities to protect these clients make decisions on their behalf. Counselors intend to keep the information discussed during a therapy session confidential and pledge not to divulge any information without their client's prior permission. As for clients, counselors must inform them entirely of their moral and legal rights to confidentiality and data privacy. With limited restrictions to confidentiality, such as the revelation of dangerous behavior, counselors should assure their clients that they can open up and chat in confidence.

\section{CONCLUSION}

This study has created a basis from which future research may derive. The findings suggest that the counselors need to explore what the new generation understands more. The new generation is very different from what counselors experienced because of the unique personalities and behavior developed during the pandemic. Moreover, even with the demanding situations (i.e., the pandemic) and misunderstandings, both 
counselors and students understand because of the different alternatives used. As long as communication is present on both sides, solving and tackling upcoming challenges becomes more accessible.

This qualitative study aims to determine the experiences of guidance counselors in the new environment as they meet the unique needs and problems of the new generation-the counselors' methods in significant understanding influence personal and professional relationships with the clients. Every counselor may have different approaches and theories towards their goal, giving them a better experience of problems and difficult situations. Guidance counselors train to consider the children's developmental and emotional levels and modify their programs to fit all clients' requirements.

As a result, clients benefit from the numerous counseling strategies provided by the school counselor and strive to solve the remaining problems. Exposing and developing an adaptation to the new environment and progressing further generates new ideas and methods for counselors to understand. Over and above that, the purpose of why counselors exist is to guide their clients and spread awareness while maintaining their ethics and focusing on their protocols and approaches. There are many procedures and guidelines to how counselors work and manage their time. Lastly is the way a counselor proceeds with their guidance follows conditions and requires coordination between the counselor and client to avoid miscommunication.

Despite the challenges of what guidance counselors face during this pandemic, they still strive to assist their clients in the possibility that some ideas and theories are left unnoticed. Researchers may utilize this study as they identify additional prospects any way possible with their methods featured in this study; there is still a significant limitation of this study as counselors must have years of experience to gather meaningful information. In addition, this study has shown that not all counselors have problems in the new environment because they have the adaptability in their profession; there are still some cases where they find the digital language challenging to understand. These experiences depend on how and where they handle a client's situation.

This study can be helpful in schools as it serves as an eye-opener towards the appearance of counselors within the presence of generation Z. It is beneficial as it may develop more teachings towards counseling with the aid of technology. The knowledge of the digital world and language remains one of the assets that counselors can still improve, and it is necessary to analyze more into what each generation takes part in the trends.

\section{REFERENCES}

[1] American Counseling Association (2014). ACA Code of Ethics. Obtained

https://readingcounselingservices.com/aca-code-of-ethics/

from:

[2] American School Counselor Association. (2019). ASCA National Model: A framework for school counseling programs (4th ed.). Alexandria, VA: Author.

[3] ASCA Ethics Committee (2020, September 1). School Counseling During a Pandemic. Obtained from:
https://www.schoolcounselor.org/Magazines/September-October-202 0/School-Counseling-During-a-Pandemic

[4] Bastemur, S., Dursun-Bilgin, M., Yildiz, Y., \&amp; Ucar, S. (2016, February 9). Alternative therapies: New approaches in counseling. Procedia - Social and Behavioral Sciences. Retrieved December 13, 2021, from

https://www.sciencedirect.com/science/article/pii/S18770428160016 09.

[5] British Association for Counselling and Psychotherapy. (2018). Ethical Framework for the Counselling Professions. Retrieved from https://www.bacp.co.uk/media/3103/bacp-ethical-framework-for-thecounselling-professions-2018.pdf

[6] Boddy, C.R.. (2016). Sample size for qualitative research. Qualitative Market Research: An International Journal. 19. 426-432. 10.1108/QMR-06-2016-0053.

[7] Boudreau. (2020, September 18). School Counselors and COVID-19. Harvard Graduate School of Education. https://www.gse.harvard.edu/news/uk/20/09/school-counselors-and-c $\underline{\text { ovid-19 }}$

[8] Cleveland, A., Sharp, S. (2019). 50+ tech tools for school counselors: How to be more engaging, efficient, and effective. Thousand Oaks, CA: Corwin

[9] Chibbaro, J. S., Ricks, L., \& Lanier, B. (2019). Digital Awareness: A Model for School Counselors. Journal of School Counseling, 17(22), n22.

[10] Demir, M., \& Can, G. (2015). Counseling and guidance understandings of guidance teachers and their attitudes towards counseling and guidance. Egitim Ve Bilim, 40(179) Retrieved from: https://search-proquest-com.eres.qnl.qa/scholarly-journals/counseling -guidance-understandings-teachers-their/docview/1682152295

[11] Feller, C.P. and Cottone, R.R. (2003), The Importance of Empathy in the Therapeutic Alliance. The Journal of Humanistic Counseling, Education and Development, 42: 53-61. https://doi.org/10.1002/j.2164-490X.2003.tb00168.x

[12] Flum, H., \& Kaplan, A. (2006). Exploratory orientation as an educational goal. Educational Psychologist, 41(2), 99-110.

[13] Gallo, L. L., Rausch, M., Smith, C. K., Wood, S. (2016). School counselors' experiences working with digital natives: A qualitative study. Professional School Counseling, 20 doi:10.5330/1096-2409-20.1.14

[14] Gibson, R. L., \& Mitchell, M. H. (2003). Introduction to Counseling and Guidance. Upper Saddle River, NJ: Merrill/Prentice-Hall.

[15] Giorgi, A. (2005). Remaining challenges for humanistic psychology. Journal of Humanistic Psychology, 45(2), 204-216. Obtained from https://www.academia.edu/36545615/Humanistic_psychology_and_t he_transformation_of_psychological_science_and_practice

[16] Han, J. (2013). Emotion in motion - A study on representation of sculpture and body form by the theme of human emotion in motion (Order No. 1543737). Available from ProQuest Dissertations \& Theses Global. (1431981539). Retrieved from https://search-proquest-com.eres.qnl.qa/dissertations-theses/emo tion-motion-study-on-representation-sculpture/docview/1431981539

[17] Hayden, L., Poynton, T., \&amp; Sabella, R. A. (2008, November) School counselors' use of technology with in the ASCA National Model's Delivery System. ResearchGate. Obtained from: https://www.researchgate.net/publication/287417135_School_counse lors'_use_of_technology_with_in_the_ASCA_national_model's_deliv ery_system.

[18] Heyden, S. M. (2011). Counseling children and adolescents Belmont, CA: Brooks/Cole. https://wikieducator.org/images/a/a7/Guidance \%26 counselling ser vices.pdf 


\section{Connecting Digitally with the Gen ' $Z$ ': \\ A Phenomenological Study on the Challenges of School Counselors amidst the Pandemic}

[19] Holcomb-Mccoy, C.C. (2004). Assessing the Multicultural Competence of School Counselors: A Checklist. Professional school counseling, 7 ,

178. https://www.semanticscholar.org/paper/Assessing-the-Multicult ural-Competence-of-School-A-Holcomb-Mccoy/030964c13ba112bb0 b64c866a88a2594f3919b18

[20] Holmes, C.M. \& Kozlowski, K.A. (2016). A group counseling collaboration model: Support for virtual high school students. In Ideas and research you can use: VISTAS 2016. Retrieved from

http://www.counseling.org/knowledge-center/vistas

[21] Julius, Andre \& Fahriza, Irfan \& Wulandari, Praditha. (2020). Digital literacy as a school counselor competence in the development of media in guidance services. Jurnal Penelitian Bimbingan dan Konseling. 5. 1-8. 10.30870/jpbk.v5i2.10106. Retrieved from https://www.researchgate.net/publication/347949042 DIGITAL LIT ERACY AS A SCHOOL COUNSELOR COMPETENCE IN TH E_DEVELOPMENT_OF_MEDIA_IN_GUIDANCE_SERVICES

[22] Kroger, J. (2007). Identity development: adolescence through adulthood. Thousand Oaks: SAGE.

[23] Meyers, L. (2018, April 30). \#disconnected: Why counselors can no longer ignore social media. Counseling Today. Retrieved December 22, 2021, from https://ct.counseling.org/2018/04/disconnected-why-counselors-can-n o-longer-ignore-social-media/

[24] Position statements, The School Counselor and Student Safety and the Use of Technology. ASCA Position Statements - American School Counselor Association (ASCA). (2021). Retrieved November 16, 2021, from https://www.schoolcounselor.org/Standards-Positions/Position-State ments/ASCA-Position-Statements

[25] Position statements, The School Counselor and Working with Students Experiencing Issues Surrounding Undocumented Status. ASCA Position Statements - American School Counselor Association (ASCA). (2021). Retrieved November 16, 2021, from https://www.schoolcounselor.org/Standards-Positions/Position-State ments/ASCA-Position-Statements

[26] Raypole, C. (2018, September 14). Rational emotive behavior therapy: Principles, techniques, efficacy. Healthline. Retrieved November 16, 2021, from https://www.healthline.com/health/rational-emotive-behavior-therapy

[27] Remeyni, D., Williams, B., Money, A. \& Swartz, E. (1998) "Doing Research in Business and Management: An Introduction to Process and Method" Sage Publications, p.97 https://research-methodology.net/research-philosophy/phenomenolog $\mathrm{y} / \#$ ftnref1

[28] Situmorang, D. D. B. (2020). Online/Cyber Counseling Services in the COVID-19 Outbreak: Are They Really New? Journal of Pastoral Care \& Counseling: Advancing Theory and Professional Practice through Scholarly and Reflective Publications, 74(3), 166-174. https://doi.org/10.1177/1542305020948170

[29] Steele, T.M., Nuckols, G., \& Stone, C.B. (2020). Technology Trends in School Counseling. Journal of school counseling, 18. Obtained from:

https://www.semanticscholar.org/paper/Technology-Trends-in-School -Counseling.-Steele-Nuckols/cbe4142b80e34ae4e1caf80df26a1f72d8 $\underline{5 \mathrm{a} 504 \mathrm{c}}$

[30] Smith, J., \& Gillon, E. (2021). Therapists' experiences of providing online counselling: A qualitative study. Counselling and Psychotherapy Research, 21(3), 545-554. https://doi.org/10.1002/capr.12408

[31] Torrance, Harry. (2012). Triangulation, Respondent Validation, and Democratic Participation in Mixed Methods Research. Journal of Mixed Methods Research. 6. 111-123. 10.1177/1558689812437185.
[32] Vistas. (2013). PDF. Washington; American Counseling Association. Retrieved from: https://www.counseling.org/docs/default-source/vistas/the-use-of-soci al-media-in-counselor-education.pdf?sfvrsn=370433a5 10

[33] Weissman MM, Markowitz JC, Klerman GL (2000). Comprehensive guide to interpersonal psychotherapy. New York: Basic Books. Obtained from: https://books.google.com.qa/books?hl=en\&lr=\&id=ZxuqnPWGcW8 C\&oi=fnd\&pg=PP2\&dq=related:Bjw8aQ-QnR8J:scholar.google.com /\&ots=531b6dTSsO\&sig= 7 MSprFn2U9Jv LWkzgXgnnf9w\&redir esc $=\mathrm{y} \# \mathrm{v}=$ onepage $\& \mathrm{q} \& \mathrm{f}=$ false

[34] Wigfield, A., Lutz, S. L., \& Wagner, A. L. (2005). Early Adolescents' Development across the Middle School Years: Implications for School Counselors. Professional School

Counseling. https://doi.org/10.1177/2156759X0500900206

[35] Wilczenski, F. L., \& Coomey, S. M. (2006). Cyber-Communication: Finding its Place in School Counseling Practice, Education, and Professional Development. Professional School Counseling. https://doi.org/10.1177/2156759X0500900404 